\title{
Performance of Geosynthetic-Reinforced Soils Under Static and Cyclic Loading
}

\author{
Mabrouk Touahmia \\ College of Engineering, Department of Civil Engineering \\ University of Hail \\ Hail, Saudi Arabia \\ m.touahmia@uoh.edu.sa
}

\begin{abstract}
This paper investigates and discusses the composite behavior of geosynthetic reinforced soil mass. It presents the results of a series of large-scale laboratory tests supported by analytical methods to examine the performance of geogrid reinforcement subjected to static and cyclic pullout loading. The testing equipment and procedures used for this investigation are outlined. The results show that geosynthetic reinforcement can mobilize great resistance to static pulling load under high confining pressures. The reinforcement exhibits gradual deformation under cyclic loading showing no sign of imminent pullout failure for all levels of applied loads. In general, the results demonstrate that geosynthetic can be used in situations where loads are non-static, although care will be required in ensuring that appropriate factors of safety are applied to control the resulting deformation. A simplified analytical model for calculating the pulling capacity of geosynthetic reinforcement is proposed.
\end{abstract}

Keywords-geosynthetics; reinforced soil; geogrid; static loading; cyclic loading

\section{INTRODUCTION}

Geosynthetic-reinforced soil technology has become a significant part of civil and environmental engineering practice all over the world due to its numerous advantages such as, costeffectiveness, easy construction and time saving. It has proven to offer sustainable alternative solutions to many soft and unstable ground problems where the use of conventional construction techniques would be restricted or significantly expensive. Various types of geosynthetic products have found several aspects of earthwork applications such as in earth retaining structures, steep slopes, roadway embankments, railway tracks, load-bearing foundations, and subsurface drainage. The inclusion of geosynthetic reinforcement in soil mass improves the shear resistance of the soil due to the interlocking of the soil particles with the reinforcement apertures, thereby improving its structural capability. Under applied external load the reinforcement mobilizes resistance along its length according to the laws of bond and bearing. The transfer of stress between soils and various soil reinforcement systems involves two basic mechanisms: frictional resistance and/or passive resistance. A great deal of research has been undertaken to understand the interaction mechanisms and the operational behavior of the geosynthetic-soil composite material [1-3]. However, design methodologies used for geosynthetic reinforced soil systems have remained variable and sometimes confusing. Up to now, there are no uniform standards and specifications for reinforced soil systems, and in fact, there are different design criteria and procedures for each system. Most reinforced soil structures have been designed using limit equilibrium methods, which are generally considered to be very conservative [4-6]. These designs consider the peak strengths of the materials without regard to the strain compatibility between the two dissimilar materials and treat all types of loads as pseudo static loading [7]. To date, engineers are faced with many uncertainties regarding the selection of appropriate types of reinforcement and the evaluation of design parameters for these systems.

Geosynthetic reinforced soil systems are frequently subjected not only to static loads but also to non-static loads such as repeated or cyclic loads. Such loading conditions can result from traffic loads on reinforced embankments and bridge abutments, pavements supported by reinforced soil retaining walls, reinforced earth structures subjected to significant tidal variations or to extremes temperature daily. The effect of cyclic loading on the interaction behavior of soil-geosynthetic systems has not yet been sufficiently addressed in research. It is considered however that the development of any reliable design methodology for geosynthetic reinforced soil systems will require a thorough understanding of the interaction mechanism and failure characteristics of the soil-geosynthetic composite material under different types of loading conditions.

The main objective of this paper is to examine the pullout behavior of geogrid reinforcements under static and slow cyclic loading conditions. A large amount of laboratory test data has been generated in order to investigate the effects of various parameters on the interactive behavior of the composite material. The mechanics of load mobilization and reinforcement deformation under loading and unloading modes has been analyzed.

\section{EXPERIMENTAL INVESTIGATION}

The schematic diagram of the large-scale pullout apparatus used for the purpose of this experimental program is shown in 
Figure 1. It consisted basically of a rigid soil container of inside dimensions $4.0 \mathrm{~m} \times 0.3 \mathrm{~m} \times 0.3 \mathrm{~m}$, a horizontal pullout loading system with the capacity to apply either static or slow cyclic loads to the reinforcement and a normal pressure setup.

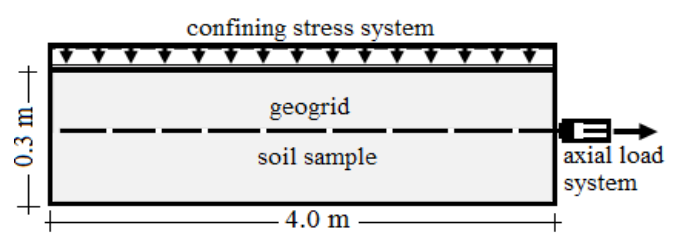

Fig. 1. Pull-out test device.

The static loading tests were carried out by adding dead weights to the load hanger situated at the rear end of the container and connected to the reinforcement. The cyclic loads were applied through lifting and releasing a moveable rider with weights on it by a reciprocating electrical motor. The repeated cycles of loading changed every 20 seconds between an upper and a lower load level to give a square-shaped pattern. The confining stress, which could be controlled from 0 to 300 $\mathrm{kN} / \mathrm{m}^{2}$, was applied to the top of the soil sample via a pressure plate loaded through a water bag. A data acquisition system was used to record the collected test results. The soil used in this investigation was a uniformly graded dry sand of medium size. The properties of the sand are given in Table I.

TABLE I. SOIL SAMPLE PROPERTIES

\begin{tabular}{|c|c|}
\hline Property & Value \\
\hline Specific gravity & 2.67 \\
Coefficient of uniformity & 1.86 \\
Coefficient of curvature & 0.84 \\
Maximum dry density $(\mathrm{Mg} / \mathrm{m} 3)$ & 1.78 \\
Minimum dry density $(\mathrm{Mg} / \mathrm{m} 3)$ & 1.42 \\
Maximum void ratio & 0.87 \\
Minimum void ratio & 0.49 \\
Friction angle & 30.4 \\
\hline
\end{tabular}

The soil samples were prepared by raining method to a targeted relative density of $53 \%$. To obtain a uniform density throughout the filling operation, the sand was poured into the pullout box from a constant height of $0.35 \mathrm{~m}$ and placed in 0.05 $\mathrm{m}$ thick equal layers. The reinforcements used in this investigation were formed by cutting Tensar SR2 geogrid into a row of two ribs in width and 35 bars length (approximately 4 $\mathrm{m})$. Special axial movement gauges were provided at 5 locations along the reinforcement to measure the axial strains with applied load. The test specimen was located at the midheight of the soil sample and connected to the loading levels system with a special clamp. The applied cyclic load levels were expressed as a percentage of the index load $(P I=3.25$ $\mathrm{kN}$ ) of the geogrid, defined as the ultimate rupture load of an identical ( 4 bars $x 2$ ribs) geogrid element in air.

\section{STATIC LOAD TEST RESUlT}

A series of static pullout tests were conducted to examine the load-deformation behavior of the geogrid reinforcement and assess its mode of failure under static loading. Four levels of normal stress namely $25,50,75$ and $100 \mathrm{kPa}$ were applied to the sand surface. The embedded geogrid was subjected to a series of short-term incremental axial pullout loads of 0.196 $\mathrm{kN} / 5 \mathrm{~min}$. The collected data from the pullout tests includes the variation of the geogrid deformation with the applied load at the frontal end and along the reinforcement length. All tests were performed till the total pullout displacement of the geogrid reaches a maximum of $40 \mathrm{~mm}$, which represents $1 \%$ of the reinforcement length. Figure 2 shows the pulloutdisplacement relationships of the geogrid reinforcement under different confining stresses $\left(\sigma_{n}\right)$.

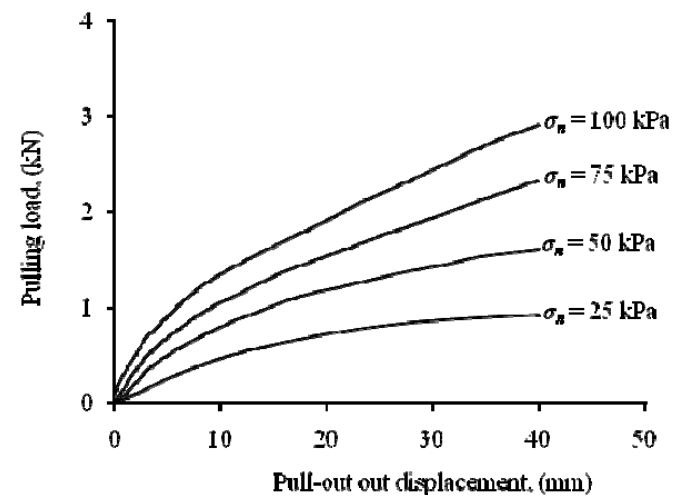

Fig. 2. Load-displacement relationships of the geogrid.

The general pattern of these relationships is characterized by a continuous increase in the geogrid movement with increase in applied loads showing no pullout failure from the beginning till the end of the test. No peak load could be observed with the system of loading used, and the relationship between load and deformation became linear at larger displacements. The surcharge pressure was found to have a great effect on the deformation of the geogrid. As shown, the reinforcement mobilized greater resistance to pulling loads when the surcharge pressure increased and that is clearly visible under the high loading increments. This resistance to pullout of the reinforcement is offered by two main components, the frictional resistance provided mainly by the longitudinal members of the geogrid, and the passive-bearing resistance provided by the transverse members of the geogrid.

The recorded movements along the length of the geogrid reinforcement for different applied pullout loads are given in Figure 3. As shown, the total deformation of the reinforcement consists only of an extension of the front part of the reinforcement and neither slip nor extension along the rear segment of the geogrid length was observed. This means that the applied load was mobilized over the front part of the strip only, the parts towards the distal end being unstrained. The magnitude of deformation along the geogrid is highly dependent on the applied confining pressure, with higher surcharge pressures resulting in very small or no load being transmitted to the rear part of reinforcement. This observation would indicate that unless a very low confining stress be used it would be impossible to pull out the geogrid reinforcement. Consequently failure of the geogrid by rupture appears to be an easier mode. 


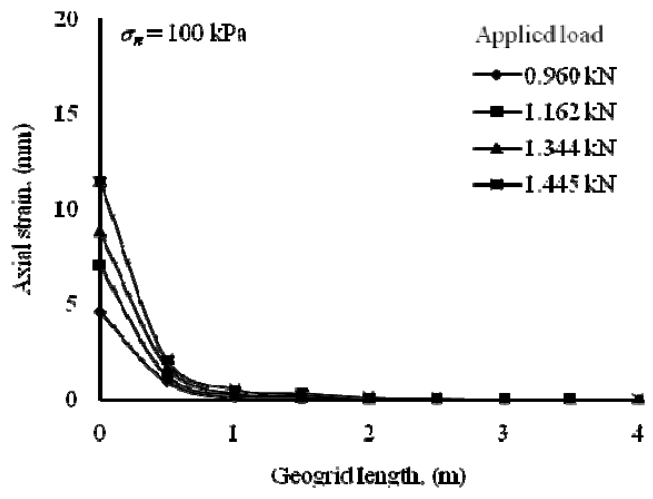

Fig. 3. Axial deformation along the geogrid.

\section{CYCLIC LOAD TEST RESULT}

A large amount of cyclic loading tests has been performed in order to obtain a better understanding of the effect of cyclic loading on the pullout behavior of geosynthetic soil reinforcements. A wide range of loading levels and amplitudes was chosen to examine the pullout behavior and the failure pattern of the geogrid under such loading condition. In some cases the tests were taken to 100000 load cycles while the confining pressure was kept constant at $100 \mathrm{kPa}$ for comparison purpose. The cumulative movement of the reinforcement versus the number of loading cycles is presented in Figure 4.

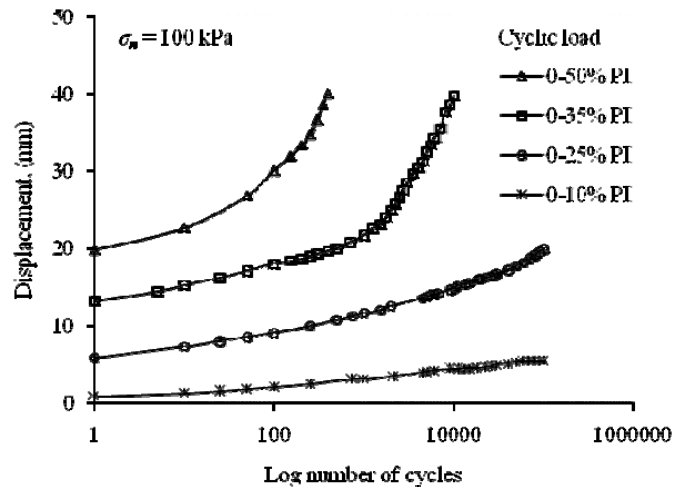

Fig. 4. Displacement-log number of load cycles relationships.

As shown, the general behavior of the reinforcement was characterized by a continual increase in displacements with ian ncrease in loading cycles from the starting till the end of the test. Despite the large number of load repetitions none of the tested strips failed by pulling out. All the geogrid reinforcements were gradually pulling through the soil, as the number of load cycles increased, showing no sign of absolute failure.

The gradual deterioration of the geogrid performance can be attributed primarily to the bearing resistance developed by the bars of the grid which acted as a series of anchors and, secondly, to the high extensibility characteristic of the geogrid material. It can be noted that the behavior of the geogrid reinforcement was highly dependent upon the magnitude of the cyclic load, with higher amplitude causing a rapid deterioration of the reinforcement performance. The life span of the geogrid was prolonged from 400 cycles to a nearly 10000 cycles by a reduction in the load amplitude from $50 \%$ PI to $35 \%$ PI and the reinforcement sustained more than 105 cycles when it was subjected to a loading amplitude of $25 \% \mathrm{PI}$. With further lowering of the loading amplitude to $10 \% P I$, the geogrid remained stable for over 105 cycles showing a nearly linear relationship between the reinforcement frontal displacement and the log number of load cycles.

For further examination of the geogrid failure mechanism, the rate of movement per cycle is plotted against the number of load cycles on log-log scale. As shown in Figure 5, all the curves showed a similar trend which is characterized by a linear relationship of a decreasing rate of movement with increasing number of load cycles from the beginning till the end of the testing period. Such behavior indicates no sign of imminent pullout failure despite the large number of load cycles and the large amount of displacement.

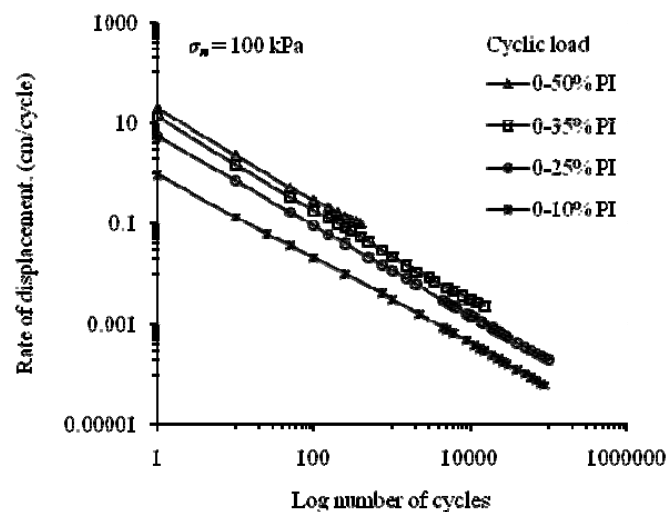

Fig. 5. Rate of displacement-log number of load cycles relationships.

The effect of the loading condition is further clarified in these diagrams, where different load levels gave different straight lines. An interesting feature is that the slopes of all plots are predominantly the same which indicate that the relationship is essentially independent of the stress level, and increases in stress serve only to shift the line vertically upwards. Based on this series of constant lines the frontal deformation $\delta_{N}$ of the geogrid reinforcement at $N$ number of load cycles can be expressed as:

$$
\delta_{N}=e^{\left[\delta_{i}+\left(s_{p}+1\right) \log N\right]}
$$

where $\delta_{i}$ is the deformation after one cycle and $s_{p}$ is the slop of the deformation-number of cycles relationship.

The total deformation at any section along the geogrid and its variation with load reversals is shown in Figure 6. These movements were expected to comprise two components as the number of cycles increases, the slip movement of the reinforcement together with the extension of the geogrid material. However, as can be seen, the total deformation consists only of an extension of the front segments and neither slip nor extension along the distal end of the reinforcement was 
observed even at the highest loading level 0.5 PI. This observation would indicate that no load was transmitted to the lattermost half of the reinforcement which remained unstressed during all the cyclic loading tests.

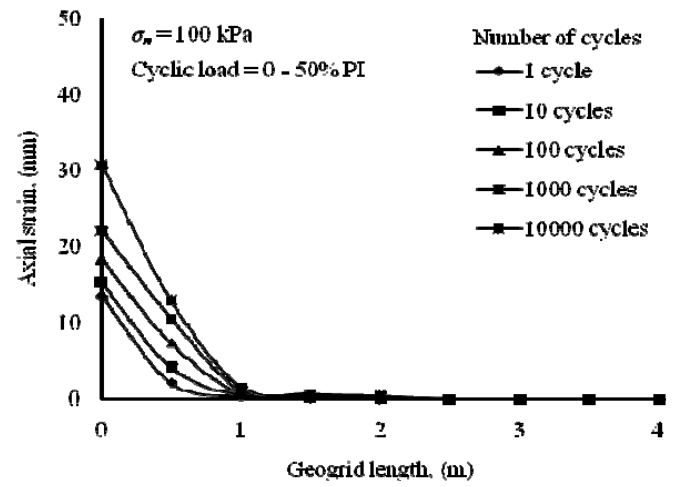

Fig. 6. Change in axial strain with number of load cycles.

It can be noted that the load was mobilized over the front part of the strip only, the parts towards the distal end being unstrained. This observation would indicate that unless a very low confining stress be used it would be impossible to pull out the geogrid. Consequently, failure of this reinforcement by rupture appears to be an easier mode.

\section{ANALYTICAL MODEL}

Due to geometrical and mechanical characteristics, geogrid can only carry tensile stresses through contact zone and anchorage members. Under applied external load the reinforcement mobilizes progressively resistance along its length according to the laws of band and bearing. Thus, the pullout resistance of the geogrid reinforcement would consist of two parts; (i) frictional resistance due to skin friction $\left(F_{a}\right)$ and (ii) passive-bearing resistance against traverse members $\left(F_{b}\right)$. The overall pullout capacity of the geogrid $\left(T_{m z x}\right)$ can be computed as:

$$
T_{\max }=F_{a}+F_{b}
$$

The frictional resistance is offered mainly by the longitudinal members due to interfacial shearing between soil and the geogrid surface and can be expressed as:

$$
F_{a}=2 B^{\prime} \sigma_{n} \int_{0}^{L^{\prime}} \tau_{a}(x) d x
$$

Where $\sigma_{n}$ the applied normal pressure at the soil-reinforcement interface, $\tau_{\alpha}(x)$ the soil adhesion at the interface and $B^{\prime}$ and $L^{\prime}$ are the reinforcement effective width and length.

The passive-bearing resistance results from the interlocking of the soil at the geogrid apertures, which are normal to the pullout direction. The transverse members of a grid reinforcement can be considered analogous to a series of strip footings in succession which have been rotated through $90^{\circ}$ to the horizontal and pulled through the soil. The maximum bearing stress can be calculated by invoking the theory of bearing capacity [8-9] which can be expressed in cohesionless soil as:

$$
F_{b}=n \sigma_{n}\left[e^{\left(\frac{\pi}{2}+\phi\right) \tan \phi} \tan \left(45+\frac{\phi}{2}\right)\right]
$$

where $\mathrm{n}$ is the number of transverse members in the geogrid and $\phi$ is the frictional angle for soil. The total pullout resistance can be then expressed in general form as:

$$
T_{\max }=2 B \sigma_{n} \int_{0}^{L^{\prime}} \tau_{a}(x) d x+n \sigma_{n}\left[e^{\left(\frac{\pi}{2}+\phi\right) \tan \phi} \tan \left(45+\frac{\phi}{2}\right)\right]
$$

The experimental results showed that as the reinforcement is loaded the resistance available at the soil-reinforcement interface is gradually mobilized along the reinforcement surface. This means that at an applied load $\mathrm{T}$ adhesion is mobilized over a part of the reinforcement length only, the remaining part remains unloaded. At some average adhesion the strength of the interface is mobilized and failure migrates along a distance $\mathrm{x}$ of the reinforcement length. Increase of $T$ by $d T$ causes an additional displacement $d \Delta$ of the reinforcement. This incremental displacement maybe expressed by:

$$
d \Delta=\frac{d F_{a}+d F_{b}}{E} x
$$

where $d F_{a}$ is the additional frictional load over the length $x$, $d F_{b}$ is the load taken by the bearing members, and $E$ is a constant which depends on the reinforcement stiffness and properties.

Putting $d F_{a}=k d F_{b}$ and rearranging (5) gives:

$$
d \Delta=\frac{F_{a}}{2 B^{\prime} \sigma_{n} \tau_{a} E}\left(1+\frac{1}{k}\right) d F_{a}
$$

Integration of (6) yields the displacement of the geogrid reinforcement $\Delta$ as:

$$
\Delta=\frac{F_{a}^{2}}{4 B^{\prime} \sigma_{n} \tau_{a} E}\left(1+\frac{1}{k}\right)
$$

The unloading of the reinforcement may be studied by incrementally adding a reverse or negative loads until the gross effect is that of zero external applied load. Thus, the mechanics of unloading are similar to those described for the loading case, except the adhesion resistance is no longer $\tau_{\alpha}$ but $\tau \ni_{\alpha}$ mobilized in the reversed direction. This will cause a reverse displacement $\left(\Delta_{u}\right)$ of the reinforcement, which can be expressed by:

$$
\Delta_{u}=\frac{F_{u}^{2}}{4 B^{\prime} \sigma_{n} \tau_{a}^{\prime} E}\left(1+\frac{1}{k}\right)
$$


hence, the residual displacement $\Delta_{r}$ under zero applied load can be computed as:

$$
\Delta_{r}=\Delta-\Delta_{u}=\frac{1}{4 B^{\prime} \sigma_{n} E}\left(1+\frac{1}{k}\right)\left[\frac{F_{a}{ }^{2}}{\tau_{a}}-\frac{F_{u}{ }^{2}}{\tau_{a}^{\prime}}\right]
$$

On reloading the displacement may not equal to the original displacement which resulted on first loading unless the interface adhesion remains constant. This indicates that the act of subjecting a reinforcing element to a package of cyclic loading modifies the stress state along the reinforcement which leads to a progressive pullout of the reinforcement.

\section{CONCLUSION}

This study investigated the composite behavior and performance of geosynthetic reinforced soil mass under static and cyclic loading. A series of pullout tests were conducted on geogrid embedded in a uniformly graded dry sand of medium density under different vertical confining pressures. The tests provide a better understanding of the interaction mechanism and failure mode of soil-geogrid composite systems that can be used for validation of analytical models. The findings demonstrate that geosynthetics have great potential to be used in situations where loads are non-static, although care will be required in ensuring that appropriate factors of safety are applied to control the resulting deformation. The study brings the following conclusions for geogrid reinforcement:

- Under static loading the geogrid mobilizes greater resistance to pulling load under higher confining pressure.

- Under cyclic loading the geogrid exhibits a gradual deformation showing no sign of imminent pullout failure for all levels of applied load. The accumulation of deformation increases with increase in the number of load repetitions and load amplitude.

- The deformation of the geogrid consists of an extension of the front part of the reinforcement only and neither slip nor extension along the rear segment was observed.

- Based on the test results analysis, a simplified analytical model for calculating the pulling capacity and deformation of geogrid reinforcements under loading and unloading conditions is proposed.

\section{ACKNOWLEDGMENT}

The research reported herein was funded by the Deanship of Scientific Research at the University of Hail, Saudi Arabia, under the contract (0150215). The author would like to extend his gratitude to the Deanship of Scientific Research and to the College of Engineering at the University of Hail for providing all facilitations required for this research.

\section{REFERENCES}

[1] M. B. Hossain, M. Z. Hossain, T. Sakai, "Interaction properties of geosynthetic with different backfill soils", International Journal of Geosciences, Vol. 3, No. 5, pp. 1033-1039, 2012
[2] J. P. Giroud, "Quantification of geosynthetic behavior", Geosynthetics International, Vol. 12, No. 1, pp. 2-27, 2015

[3] E. M. Palmeira, "Soil-geosynthetic interaction: modelling and analysis", Geotextiles and Geomembranes,Vol. 27, No. 5, pp. 368-390, 2009

[4] J. G. Zornberg, D. Leshchinsky, "Comparison of international design criteria for geosynthetic-reinforced soil structures" in Landmarks in Earth Reinforcement, Ochiai et al. (Eds), Vol. 2, pp. 1095-1106, 2003

[5] K. H. Yang, P. Utomo, T. L. Liu, "Evaluation of force-equilibrium and deformation based design approaches for predicting reinforcement loads within geosynthetic reinforced soil structures", Journal of GeoEngineering, Vol. 8, No. 2, pp. 41-54, 2013

[6] M. P. Pinho-Lopes, A. M. Paula, M. L. Lopes, "Soil-geosynthetic interaction in pullout and inclined-plane shear for two geosynthetics exhumed after installation damage", Geosynthetics International, Vol. 23, No. 5, pp. 331-347, 2016

[7] A. McGown, "The behaviour of geosynthetic reinforced soil systems in various geotechnical applications", $2^{\text {nd }}$ European Conference on Geosynthetics, EuroGeo 2000, Bologna, Italy, 1, pp. 3-26, 2000

[8] R. A. Jewell, G. W. E. Milligan, R. W. Sarsby, D. Dubois, "Interaction between soil and geogrids", in Polymer Grid Reinforcement, Thomson Telford Limited, London, UK, pp. 18-30, 1984

[9] M. J. I. Alam, S. R. Lo, M. R. Karim, "Pull-out behaviour of steel grid soil reinforcement embedded in silty sand", Computers and Geotechnics, Vol. 56, pp. 216-226, 2014 